\title{
On the Performance of Coded Copper Wire Systems for G-fast Communications
}

\author{
Ali J. Al-Askery* and Ali Mehdi Hammadi \\ Department of Control and Automation Technical Engineering \\ Electrical Engineering Technical College/ Middle Technical University \\ Baghdad, Iraq/Phone: +964 7817569229 \\ e-mail: alialaskary1@yahoo.com
}

\begin{abstract}
Article Info
ABSTRACT

Article history:

Received Dec 25, 2018

Revised Jan 21, 2019

Accepted Feb 22, 2019

This paper aims to investigate the performance of different channel coding techniques over copper wire channels. First, Chen model is used to simulate the copper wire channel model, then the performance of LDPC coded system with DVB-S2 standard

Keywords:

Chen model copper wired channel Coding techniques is compared to the performance of turbo coded, and convolutional coded systems over wired channels. In addition, the performance of the systems under consideration is further investigated in the presence of impulsive noise (IN) with IN cancellation technique. Matlab simulations are utilized to simulate these systems for different quadrature amplitude modulations (QAM). The results have shown that LDPC coded system with large block length have outperforms the performance of the considered coding techniques.
\end{abstract}

Copyright 2019 Institute of Advanced Engineering and Science. All rights reserved.

Corresponding Author:

Dr.Ali Al-Askery

Electrical Engineering Technical College

Middle Technical University

Mobile: 009647817569229

Email: alialaskary1@yahoo.com

\section{Introduction}

Digital subscriber line (DSL) is a technology for transmitting and receiving the data over copper wire lines. Due to the expensive cost of replacing the infrastructure of the twisted pair copper wire, DSL has been evolved over the years to support the requirements of very high bit rate transmission over these lines. The G.fast is a standardization for DSL technology that supports up to $106 \mathrm{MHz}$ broad spectrum which is expected to be expanded to $212 \mathrm{MHz}$ [1]. In addition, discrete multitone (DMT) transmission converts the transmitted data into a low rate multi-carrier sequence, which makes it more convenient and efficient in the data transmission [2].

Channel coding has been used widely to improve the transmission reliability and to enhance the bite error rate (BER) performance [3-5]. In [6], turbo coded ADSL-DMT system with 64 quadrature amplitude modulation (QAM) is proposed to improve the system performance. The performance has been compared to the trellis coded modulation (TCM) to show the improvement

Journal homepage: http://iaescore.com/journals/index.php/ijeecs 


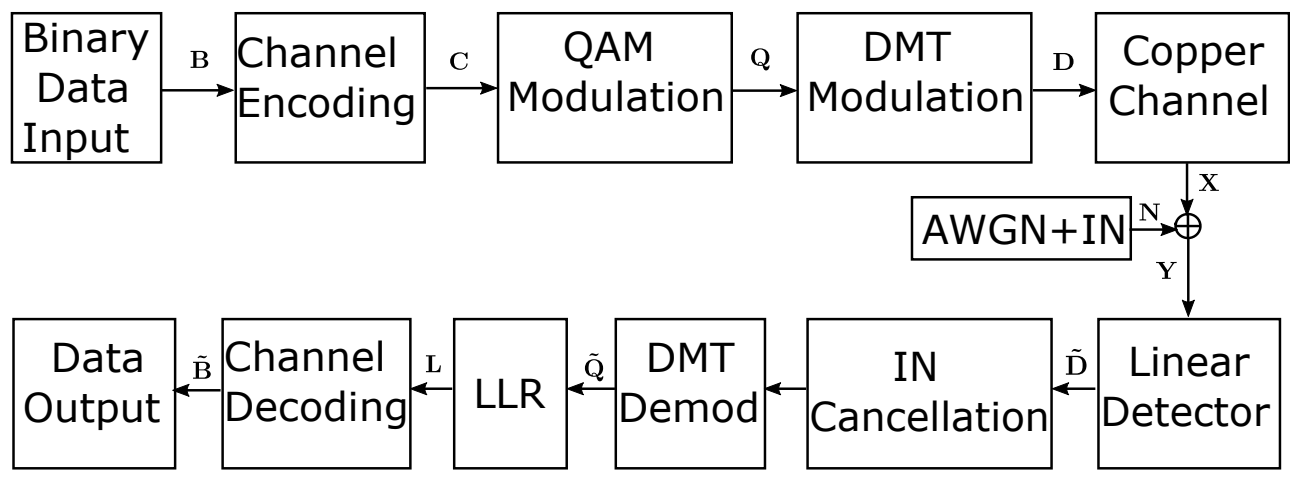

Figure 1. System model for the copper line wired system

in the coding gain. In [7], a differential evolution algorithm aided turbo channel estimation (DEA$\mathrm{CE}$ ) has been proposed for the G.fast systems to achieve near Shannon capacity limit with a reduced complexity. In addition, the performance of G.fast coded copper wire channel has been taken into consideration in [8] in the presence of impulsive noise (IN), in which, the coded system has been designed as a cascaded trellis code as an outer code with non binary Reed-Solomon (RS) code as an internal code.

Low-density parity-check (LDPC) coding has been used in [9] as an inner code for DSL system with DMT modulation, while the outer code was selected as RS code with Galois field $\mathrm{GF}\left(2^{8}\right)$. The code-word length was selected between 529 and 4489 bits with multiple M-QAM scheme, while the IN effect on the performance has not been considered. An algorithm to calculate the IN statistics has been proposed in [10] to calculate an accurate log-likelihood ratios (LLRs) for the LDPC coded DSL system. DMT modulation has been used in this paper with a precoding technique and the results have been verified utilizing the EXIT chart. On the other hand, a generator matrix with low density has been proposed in [11] which is more convenient in the video transmitting and receiving. In addition, a further extension to the proposed matrix has been demonstrated based on rearranging the matrix columns which result in a better immunity to the burst errors.

Several techniques to mitigate the IN have been taken into consideration including clipping and blanking. In [12], an IN mitigation technique has been proposed to remove the effect of the IN model of the English and German wired systems. A comparison of different IN mitigation techniques have also been provided to show the validity of the proposed technique.

In order to enable the DSL systems to support wide spectrum and support high quality video streaming, a powerful channel coding is required that improves the BER performance and increase the transmission reliability. LDPC coding with digital video broadcasting-satellite-second generation (DVB-S2) is an LDPC standard with 64800 bits code-word with irregular encoding and decoding procedure has near Shannon limit compared to other channel coding techniques $[13,14]$.

Therefore, the contribution of this paper is to investigate the performance of LDPC coded copper wire with DVB-S2 standard and comparing the system performance to other channel coding techniques such as turbo coding and convolutional coding with different M-QAM schemes. The performance of the techniques under consideration is investigated over G.fast system applications with DMT transmission and Middleton class A model for IN representation. Finally, the IN effect is mitigated from the received signal utilizing clipping technique.

This paper is organized as follows, in Section 2. the system model is presented in details, including the channel model of the wired system, Middleton class A distribution as IN model, the LLR equations are presented as part of computing the soft decoding for the received coded signal, and the sum-product algorithm (SPA) for LDPC decoder with DVB-S2 standard. The simulation and results are discussed in Section 3. While the conclusions are drawn in Section 4. 


\section{System Model}

This paper considers the G.fast system model that is illustrated in Figure 1. The binary data $\mathbf{B}$ is first generated and encoded with the relevant channel encoder to generate the codeword $\mathrm{C}$. Then the code-word is modulated with M-QAM and DMT modulator to produce the transmitted data $\mathbf{D}$ that travels over the copper line wire that is represented as Chen model [15]. At the receiver, the additive white Gaussian noise (AWGN) and the IN is added to the received signal such that

$$
\mathbf{Y}=\mathbf{H} \cdot \mathbf{D}+\mathbf{N}+\mathbf{I},
$$

where, $\mathbf{Y} \in \mathbb{C}^{1 \times K}$ and $\mathbf{D} \in \mathbb{C}^{1 \times K}$ are the received and transmitted vectors with $K$ as the length of the transmitted signal. $\mathbf{H} \in \mathbb{C}^{1 \times K}$ and $\mathbf{N} \in \mathbb{C}^{1 \times K}$ are the channel and the noise parameter vectors, respectively, and $\mathbf{I} \in \mathbb{C}^{1 \times K}$ is the IN vector. The received signal is equalized with minimum-mean squared-error (MMSE) detector such that,

$$
\mathbf{W}_{m m s e}=\mathbf{H}^{*} /\left(\left|\mathbf{H}^{2}\right|+\sigma^{2} / E s\right)
$$

where $\mathbf{H}^{*}$ is the complex conjugate of the vector $\mathbf{H}$, and $E_{s}$ is the symbol energy.

Following signal equalization, IN compensation, and DMT demodulation, the signal $\tilde{\mathbf{Q}}$ is utilized to calculate the log-likelihood ratio (LLR) for the soft decoding of the selected forward error correcting code (FECC). Finally, the decoded signal $\tilde{\mathbf{B}}$ is compared to the binary generated sequence at the transmitter to calculate the BER. It is worth mention that the channel is assumed to be known at the receiver.

\subsection{Channel Model}

As mentioned before, the channel model of this paper is based on Chen model to represent the copper wire model, which is suitable for up to $200 \mathrm{MHz}$ frequency range [16]. The attenuation constant $\alpha$ and the phase constant $\beta$ are calculated with Chen model constants $k_{1}$, $k_{2}$, and $k_{3}$, and for the frequency $f$ as

$$
\begin{aligned}
& \alpha(f)=k_{1} \sqrt{f}+k_{2} f, \\
& \beta(f)=k_{3} f .
\end{aligned}
$$

The channel parameters can be written using (3) and the cable length $L$ for a specific frequency as

$$
H=e^{-L \gamma(f)},
$$

where, the propagation constant $\gamma(f)=\alpha(f)+j \beta(f)$ and $L$ is measured in meter.

\subsection{Impulsive Noise Model}

Middleton class A distribution $[17,18]$ is used in this paper to simulate the IN of the copper wire line communication. The probability density function (PDF) of this model can be written as

$$
p(\alpha)=\sum_{\nu=0}^{\infty} \frac{e^{-A} A^{\nu}}{\nu !} \cdot \frac{e^{\left(-\frac{\alpha^{2}}{2 \sigma^{2}}\right)}}{\sqrt{2 \pi \sigma_{\nu}^{2}}},
$$

where the impulsive index is $A$, and the smallest value of $A$ will result in a very high peak, while the distribution approaches Gaussian distribution when $A$ is very high. The noise power can be calculated as $\sigma^{2}=\sigma_{G}^{2}+\sigma_{I}^{2}$, where $\sigma_{G}^{2}$ and $\sigma_{I}^{2}$ are the variance of the AWGN and the IN, respectively. The variance $\sigma_{\nu}^{2}$ can be calculated as,

$$
\sigma_{\nu}^{2}=\sigma^{2}\left(\frac{\frac{\nu}{A}+\Gamma}{1+\Gamma}\right)
$$

and $\Gamma=\sigma_{G}^{2} / \sigma_{I}^{2}$. Zhidkov compensation algorithm is used in this paper to remove the IN effect as shown in [8]. 


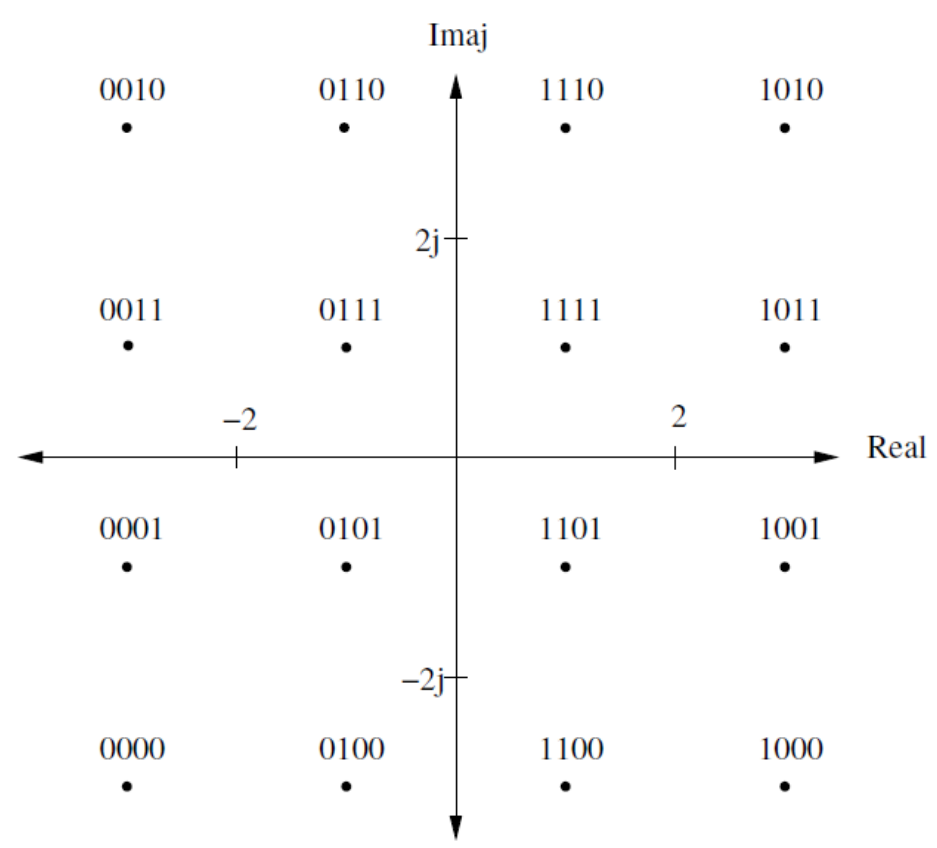

Figure 2. Constellation map of the 16-QAM scheme.

\subsection{LLR Calculations}

The LLRs for the system under consideration can be calculated based on the QAM constellation index such that $[14,19]$,

\subsubsection{4-QAM Scheme}

The LLR of this constellation can be calculated as,

$$
\begin{aligned}
& L_{0}=\ln \left(\frac{p\left(\left.N\right|_{D^{I}=1}\right)}{p\left(\left.N\right|_{D^{I}=-1}\right)}\right), \\
& L_{1}=\ln \left(\frac{p\left(\left.N\right|_{D^{Q}=1}\right)}{p\left(\left.N\right|_{D^{Q}=-1}\right)}\right),
\end{aligned}
$$

where $L_{0}, L_{1}$ are the LLR of the two bits $B_{0}, B_{1}$, respectively, and $p(N)$ represents the PDF of the AWGN. The superscripts $I$ and $Q$ are to indicate the in-phase and the quadrature parts of the signal.

\subsubsection{6-QAM Scheme}

The LLR of the 16-QAM constellation presented in Figure 2 can be calculated using,

$$
\begin{aligned}
L_{0} & =\ln \left(\frac{p\left(\left.N^{I}\right|_{D^{I}=1}\right)}{p\left(\left.N^{I}\right|_{D^{I}=-1}\right)}\right), \\
L_{1} & =\min \left(\ln \left(\frac{p\left(\left.N^{I}\right|_{D^{I}=-1}\right)}{p\left(\left.N^{I}\right|_{D^{I}=-3}\right)}\right), \ln \left(\frac{p\left(\left.N^{I}\right|_{D^{I}=1}\right)}{p\left(\left.N^{I}\right|_{D^{I}=3}\right)}\right)\right), \\
L_{2} & =\ln \left(\frac{p\left(\left.N^{Q}\right|_{D^{Q=1}}\right)}{p\left(\left.N^{Q}\right|_{D^{Q}=-1}\right)}\right), \\
L_{3} & =\min \left(\ln \left(\frac{p\left(\left.N^{Q}\right|_{D^{Q}=-1}\right)}{p\left(\left.N^{Q}\right|_{D^{Q}=-3}\right)}\right), \ln \left(\frac{p\left(\left.N^{Q}\right|_{D^{Q}=1}\right)}{p\left(\left.N^{Q}\right|_{D^{Q}=3}\right)}\right)\right),
\end{aligned}
$$


where $L_{0}, L_{1}, L_{2}, L_{3}$ are the LLR of the 4 bits $B_{0}, B_{1}, B_{2} . B_{3}$, respectively.

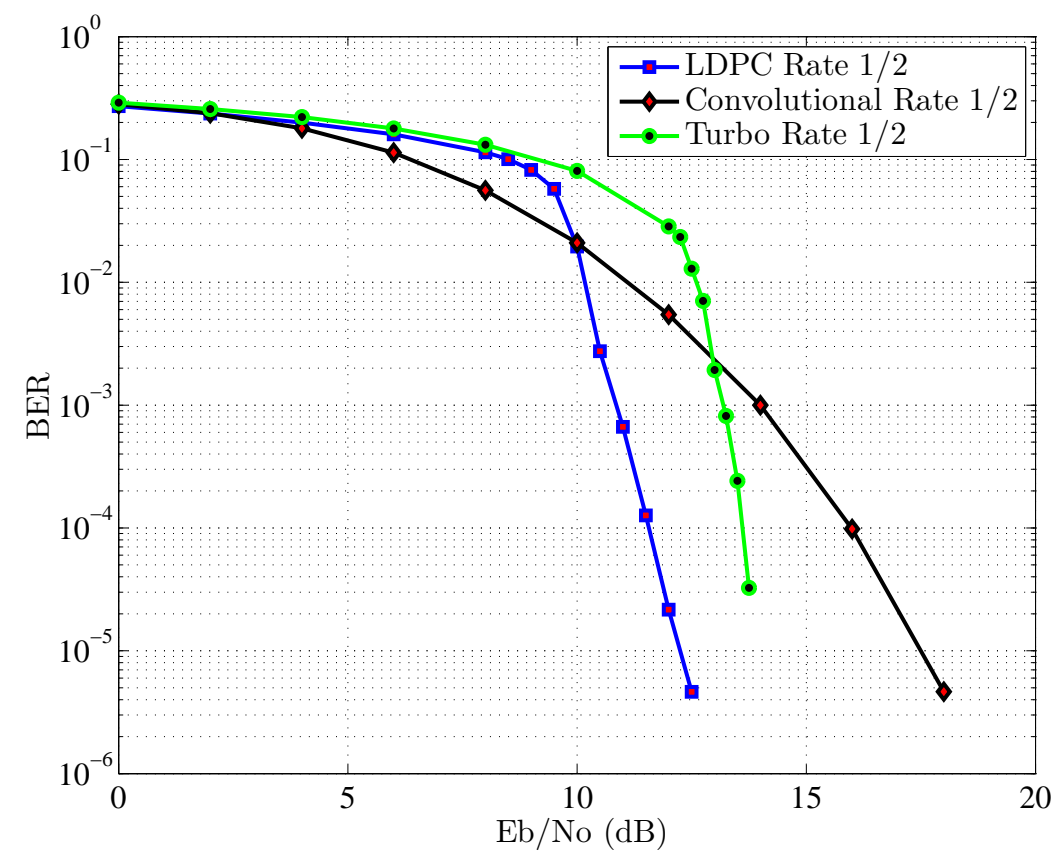

Figure 3. Simulation results at 4-QAM scheme for the turbo coded, convolutional coded and LDPC coded systems.

\subsection{LDPC Codes}

LDPC code with DVB-S2 standard and $1 / 2$ rate has a block length of $N=64800$ bits with binary data block of $k=32400$ bits. In addition, irregular LDPC codes has an improved performance compared to regular codes in which, the BER performance approaches the Shannon capacity limit closely $[20,21]$. The implementation of the LDPC decoder in this work is based on the efficient sum-product algorithm (SPA) that have been proposed in [22].

First, the initialization to the symbol node is the LLR equations that have been calculated in Equations (7) and (8) such that,

$$
L\left(q_{n \rightarrow m}\right)=L_{k}
$$

Next, the check to node update for each $m$ and for $n \in \mathcal{N}(m)$ is performed using,

$$
L\left(r_{m \rightarrow n}\right)=2 \tanh ^{-1}\left(\prod_{n^{\prime} \in \mathcal{N}(m) n} \tanh \left[\frac{1}{2} L\left(q_{n^{\prime} \rightarrow m}\right)\right) .\right.
$$

The third step involves updating the node to check for $n$ and for $m \in \mathcal{M}(n)$ utilizing,

$$
L\left(q_{n \rightarrow m}\right)=L_{k}+\sum_{m^{\prime} \in \mathcal{M}(n) m} L\left(r_{m^{\prime} \rightarrow n}\right) .
$$

Then, the decision on the decoded bits can be made by calculating the following equation,

$$
L\left(u_{k}\right)=L_{k}+\sum_{m^{\prime} \in \mathcal{M}(n)} L\left(r_{m^{\prime} \rightarrow n}\right),
$$

such that $u_{k}=0$ for $L\left(u_{k}\right) \geq 0$ and $u_{k}=1$ otherwise. These steps will be repeated several times until the condition $\mathbf{H} \hat{\mathbf{u}}=0$ is achieved or the maximum number of iterations is reached. 


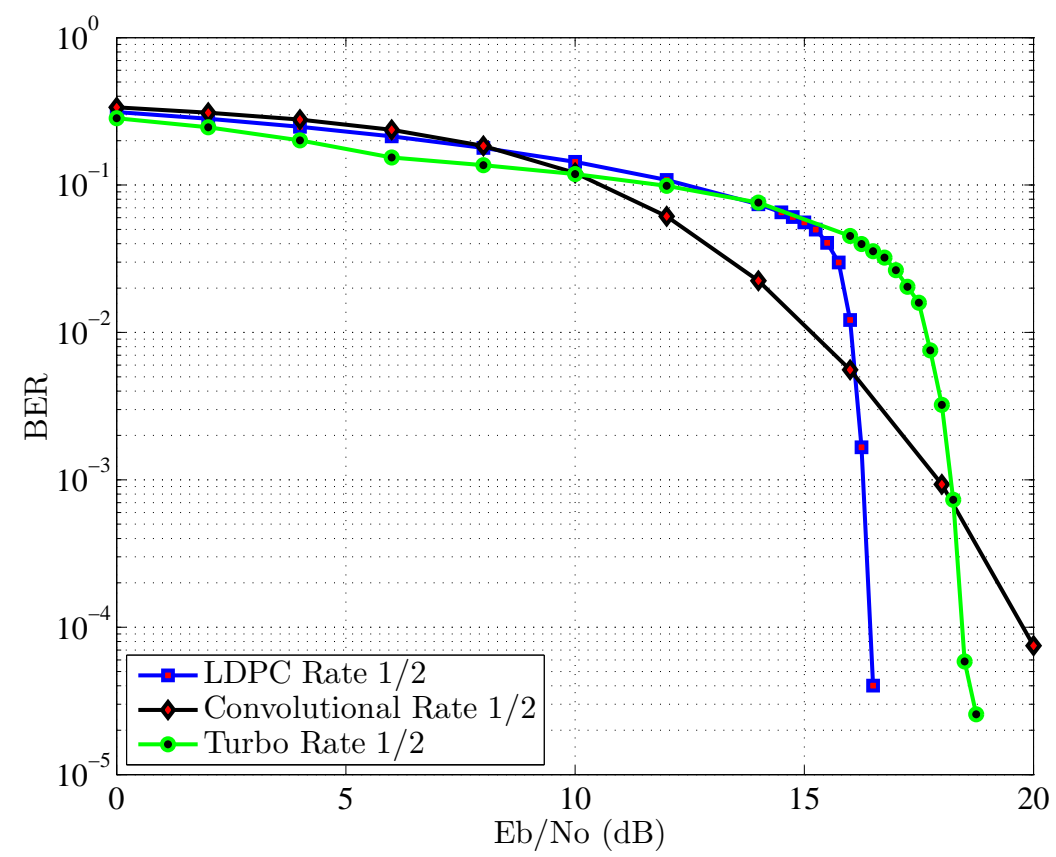

Figure 4. Simulation results at 16-QAM scheme for the turbo coded, convolutional coded and LDPC coded systems.

\section{Simulation and Results}

This section is used to illustrate and discuss the results obtained from the simulations of the wired copper system. The channel length was selected such that $L=100 \mathrm{~m}$, which is substituted in (4). Two QAM schemes have been used in this simulation which are 4-QAM and 16-QAM, respectively. MMSE detector have been used to equalize the received signal to remove the effect of the channel. The turbo decoder was $\log -M A P$ type with $1 / 2$ rate and randomly interleaved with block length 4096 bit. The eIRA LDPC decoder with rate $1 / 2$ has a length of 64800 bits.

In Figure 3, LDPC coded system with 4-QAM modulation outperforms the performance of both convolutional coded and turbo coded systems at low signal to noise ratio (SNR). It is observed that there is an improvement of $2.5 \mathrm{~dB}$ in the performance compared to the turbo coded system at $10^{-4} \mathrm{BER}$, while the improvement was almost $5 \mathrm{~dB}$ compared to the convolutional coded system.

In Figure 4, it is observed that the performance of the LDPC coded system have better performance compared to the convolutional coded and the turbo coded systems. The improvement in the BER was $2 \mathrm{~dB}$ compared to the turbo coded while it was $4 \mathrm{db}$ with respect to the convolutional coded system at $10^{-4}$ of BER.

In Figures 5 and 6 , the effect of the IN on the performance of the coded systems can be observed such that a degradation in the BER performance of almost $10 \mathrm{~dB}$ is achieved for the LDPC coded system at 4-QAM and 16-QAM schemes. However, the BER degradation was 13 $\mathrm{dB}$ for the turbo coded and convolutional coded systems. The IN compensation system have improved the performance of the LDPC and turbo coded systems with 4-QAM scheme by $6 \mathrm{~dB}$ while the improvement was $8 \mathrm{~dB}$ for the convolutional coded system. However, at 16-QAM scheme, the improvement for all the coded systems was $4 \mathrm{~dB}$. 


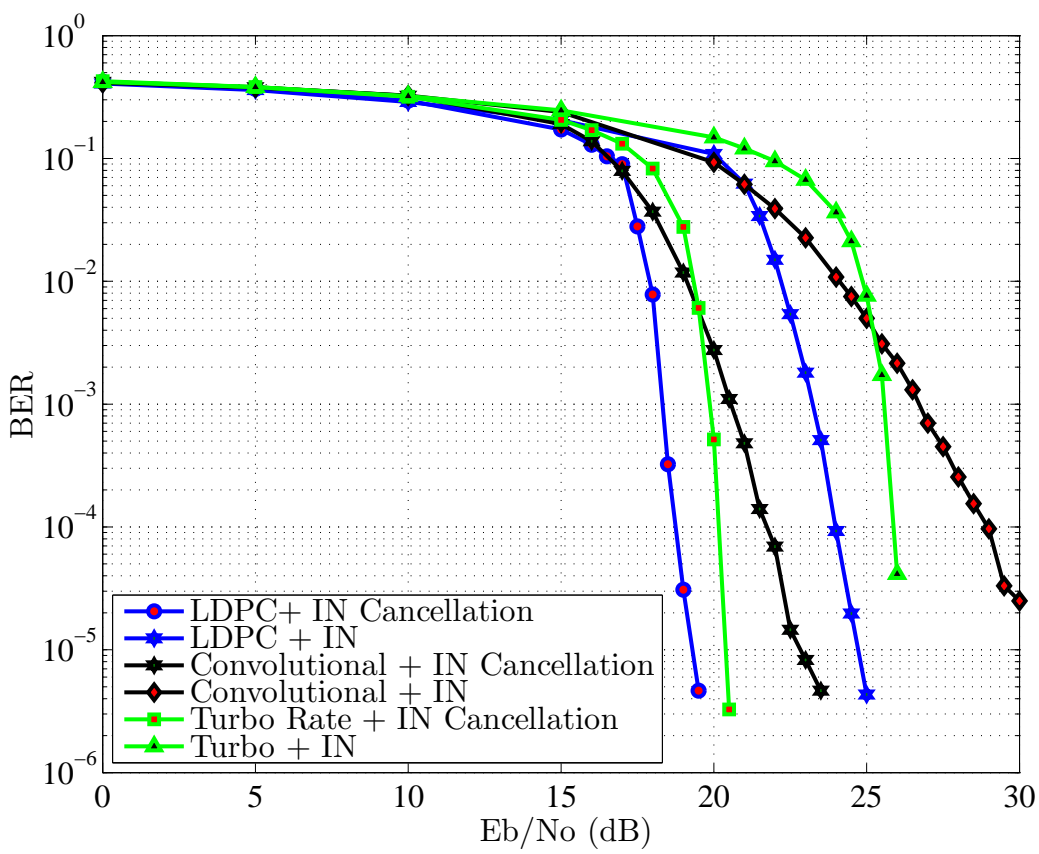

Figure 5. Simulation results at 4-QAM scheme for the turbo coded, convolutional coded and LDPC coded systems in the presence of IN.

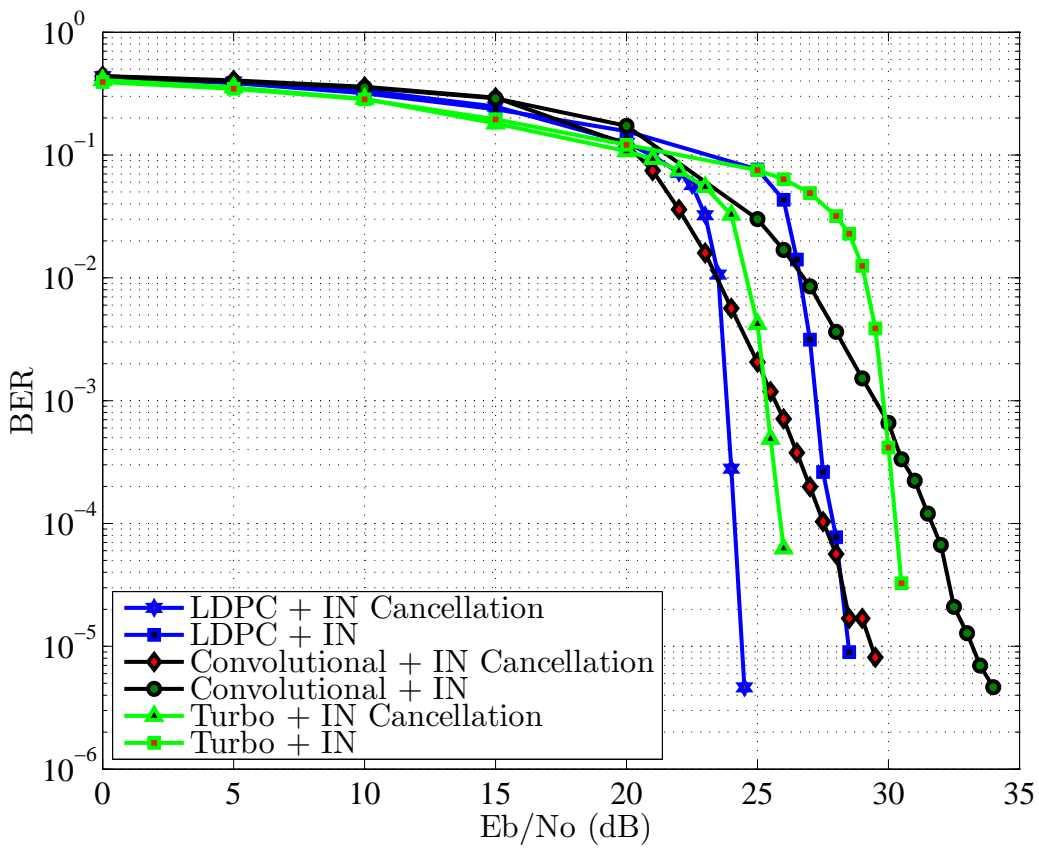

Figure 6. Simulation results at 16-QAM scheme for the turbo coded, convolutional coded and LDPC coded systems in the presence of IN. 


\section{Conclusion}

In conclusion, the performance of coded wired line systems have been examined with three channel coding techniques and two modulation indexes. The results have shown that LDPC coded systems outperforms the other coding techniques and especially at high SNR values. It is also observed that the water fall for both iterative codes are very steep, while the convolutional coded system has smooth performance curve over the SNR range due to their superior ability to detect and correct the errors. The BER degradation resulting from including the IN have been examined with Zhidkov compensation algorithm as an IN mitigation technique. As a result, the performance of the coded systems have degraded by almost $6 \mathrm{~dB}$ even after removing the effect of the IN. However, the performance of LDPC coded systems remains on the lead compared to the considered coding techniques.

\section{References}

[1] P. Odling, T. Magesacher, S. Host, P. O. Borjesson, M. Berg, and E. Areizaga, "The fourth generation broadband concept," IEEE Commun. Mag., vol. 47, no. 1, pp. 62-69, 2009.

[2] B. R. Saltzberg, "Comparison of single-carrier and multitone digital modulation for ADSL applications," IEEE Commun. Mag., vol. 36, no. 11, pp. 114-121, 1998.

[3] N. A. Khairi, A. B. Jambek, and R. C. Ismail, "Performance evaluation of arithmetic coding data compression for internet of things applications," Indonesian Journal of Electrical Engineering and Computer Science, vol. 13, no. 2, pp. 591-597, 2019.

[4] N. Telagam, S. Lakshmi, and K. Nehru, "BER analysis of concatenated levels of encoding in GFDM system using labview," Indonesian Journal of Electrical Engineering and Computer Science, vol. 14, no. 1, pp. 80-91, 2019.

[5] A. Haffane, A. H. M. Khelifi, and B. Kadri, "Convergence analysis of the unpunctured turbo trellis-coded modulation (UTTCM)," Indonesian Journal of Electrical Engineering and Computer Science, vol. 13, no. 2, pp. 447-452, 2019.

[6] L. Zhang and A. Yongacoglu, "Turbo coding for transmission over ADSL," in Proc. Int. Conf. Commun. Technol. WCC-ICCT2000., vol. 1. IEEE, 2000, pp. 124-131.

[7] J. Zhang, S. Chen, R. Zhang, A. F. A. Rawi, and L. Hanzo, "Differential Evolution Algorithm Aided Turbo Channel Estimation and Multi-User Detection for G. Fast Systems in the Presence of FEXT," IEEE Access, vol. 6, pp. 33 111-33 128, 2018.

[8] I. Al-Neami, C. T. Healy, M. Johnston, and C. Tsimenidis, "Investigation into impulsive noise techniques for a G. FAST system," in Proc. 11th Int. Sympo. Commun. Syst., Net. \& Digital Signal Process. (CSNDSP). IEEE, 2018, pp. 1-5.

[9] E. Eleftheriou and S. Olcer, "Low-density parity-check codes for digital subscriber lines," in Proc. IEEE Int. Conf. Commun., vol. 3. IEEE, 2002, pp. 1752-1757.

[10] T. Bai, C. Xu, R. Zhang, A. F. Al Rawi, and L. Hanzo, "Joint Impulsive Noise Estimation and Data Detection Conceived for LDPC-Coded DMT-Based DSL Systems," IEEE Access, vol. 5, pp. 23 133-23 145, 2017.

[11] L. Al-Jobouri, F. Casu, M. Fleury, and J. Cabrera, "Video over DSL with LDGM Codes for Interactive Applications," Computers, vol. 5, no. 2, pp. 1-18, 2016.

[12] R. Fantacci, A. Tani, and D. Tarchi, "Impulse noise mitigation techniques for XDSL systems in a real environment," IEEE Trans. Consum. Electron., vol. 56, no. 4, 2010.

[13] R. A. Carrasco and M. Johnston, Non-binary error control coding for wireless communication and data storage. John Wiley \& Sons, 2008.

[14] A. J. Al-Askery, C. C. Tsimenidis, S. Boussakta, and J. A. Chambers, "Improved coded massive MIMO OFDM detection using LLRs derived from complex ratio distributions," in Proc. 20th IEEE Int. Workshop Comput. Aided Modelling Design of Commun. Links Net., CAMAD 2015, Guildford, United Kingdom, September 7-9, 2015, 2015, pp. 64-68.

[15] D. Acatauassu, S. Host, C. Lu, M. Berg, A. Klautau, and P. O. Borjesson, "Simple and causal twisted-pair channel models for G.fast systems," in Proc. IEEE Global Commun. Conf. (GLOBECOM). IEEE, 2013, pp. 2834-2839.

[16] T. Magesacher, J. R. i Riu, M. Jakovljević, M. Loiola, P. Ödling, and P. O. Börjesson, "Limits 
of ultra-wideband communication over copper," in Proc. Int. Conf. Commun. Technol., 2006, pp. $1-4$.

[17] D. Middleton, "Statistical-physical models of electromagnetic interference," IEEE Trans. Electromagn. Compat., no. 3, pp. 106-127, 1977.

[18] T. Shongwe, A. Han Vinck, and H. C. Ferreira, "A study on impulse noise and its models," SAIEE Africa Research Journal, vol. 106, no. 3, pp. 119-131, 2015.

[19] A. J. Al-Askery, C. C. Tsimenidis, S. Boussakta, and J. A. Chambers, "Performance analysis of coded massive MIMO-OFDM systems using effective matrix inversion," IEEE Trans. Commun., vol. 65, no. 12, pp. 5244-5256, 2017.

[20] T. J. Richardson and R. L. Urbanke, "The capacity of low-density parity-check codes under message-passing decoding," IEEE Trans. Inf. Theory, vol. 47, no. 2, pp. 599-618, 2001.

[21] S.-Y. Chung, G. D. Forney, T. J. Richardson, and R. Urbanke, "On the design of low-density parity-check codes within $0.0045 \mathrm{~dB}$ of the Shannon limit," IEEE Commun. lett., vol. 5, no. 2, pp. 58-60, 2001.

[22] X.-Y. Hu, E. Eleftheriou, D.-M. Arnold, and A. Dholakia, "Efficient implementations of the sum-product algorithm for decoding LDPC codes," in Proc. IEEE Global Telecommun. Conf. GLOBECOM'01, vol. 2, 2001, pp. 1036-1036E.

\section{BIOGRAPHIES OF AUTHORS}

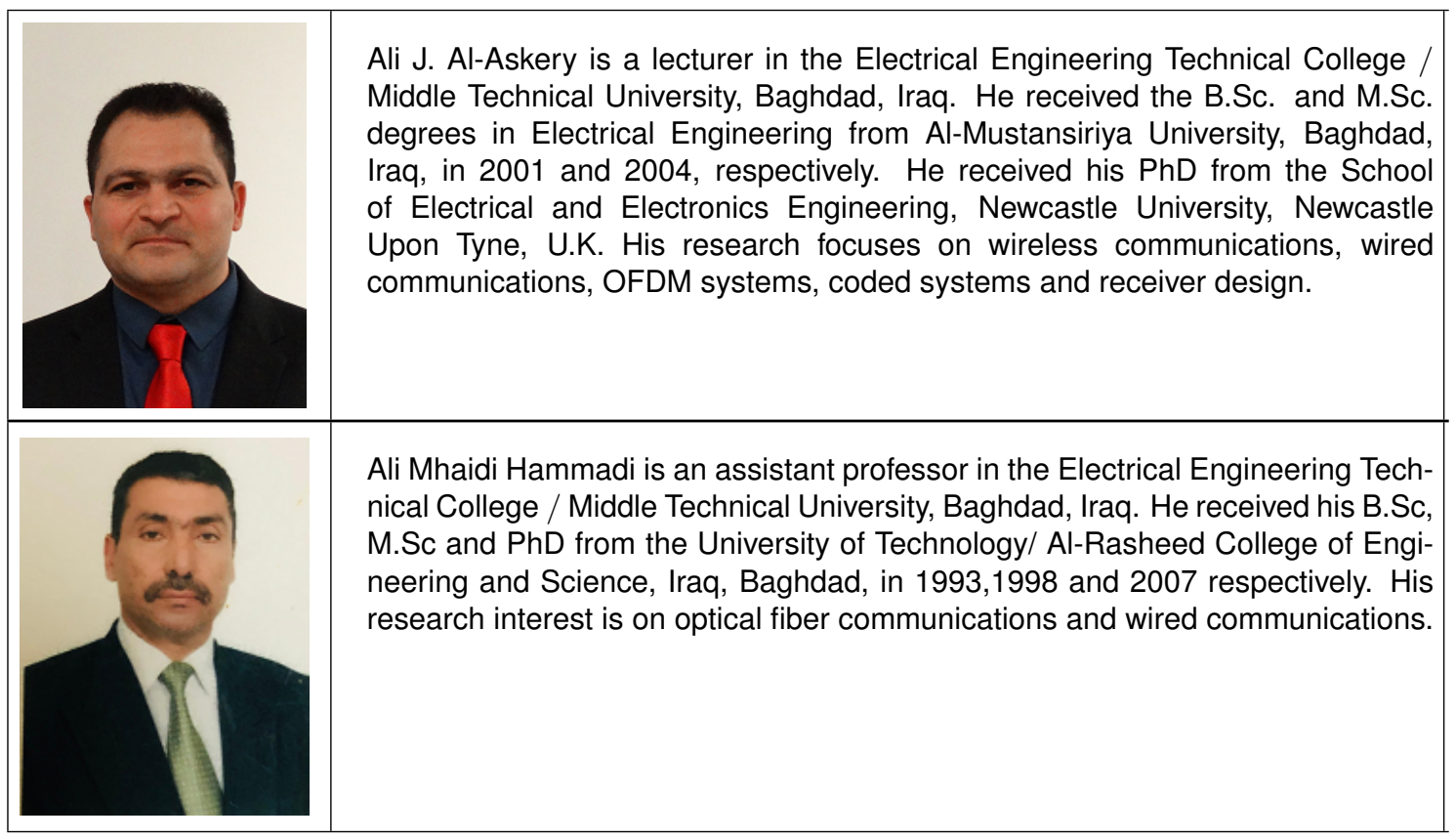

